Anuario Latinoamericano Ciencias Políticas

y Relaciones Internacionales

vol. 5, 2017

pp. $173-189$

\section{Historia y desarrollo de la ciencia política boliviana: una aproximación nacional}

DOI: 10.17951/al.2017.5.173

\section{History and development of Bolivian political science: a national approach}

\author{
Julio Ascarrunz Medinaceli \\ UNIVERSIDAD MAYOR DE SAN ANDRÉS \\ LA PAZ, BOLIVIA \\ $\triangle$ julio.ascarrunz@gmail.com
}

\title{
RESUMEN
}

El presente trabajo representa una reconstrucción descriptiva de la historia de la ciencia politica en Bolivia desde los primeros intentos de creación de la primera carrera en 1969 hasta nuestros días. Las dimensiones que se toman en cuenta para este objetivo son los programas académicos tanto de pre como de postgrado, los centros de investigación y las publicaciones periódicas, y las asociaciones profesionales/académicas presentes en el país. Tras el repaso historiográfico se pone un énfasis especial en algunos desafios a los que la disciplina debiera hacer frente si pretende seguir la institucionalización en la misma vía que sus vecinos regionales lo han estado haciendo.

PALABRAS CLAVE: ciencia política boliviana, politólogos, revistas.

\begin{abstract}
This work represents a descriptive reconstruction of the history of Bolivian political science, from the first attempts to create the first program in 1969 until the present days. The dimensions taken into account for this purpose are pre-and postgraduate academic programs, research centers, academic journals and the professional/ academic associations in the country. After the historiographic review, a special emphasis is put on some challenges that the discipline should face if it intends to continue the institutionalization in the same way that its regional neighbors have been doing.
\end{abstract}

KEYWORDS: Bolivian political science, political scientists, journals. 


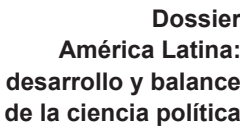

Dossie

desarrollo y balance

de la ciencia política

\section{Introducción ${ }^{1}$}

En los últimos años, en América Latina ha ido avanzando el estudio de la ciencia política desde la perspectiva del desarrollo de la disciplina. La reconstrucción de la historia disciplinar ha ayudado a entender, encaminar y reflexionar sobre los procesos de institucionalización en los diferentes países e incluso se han desarrollado diferentes investigaciones que combinan distintos casos, métodos, y enfoques.

La producción más prolífica en este campo se puede encontrar en/sobre países como Argentina (Leiras et al. 2005, Bulcourf 2012, Bulcourf y Cardozo 2013, Bulcourf et al. 2013, D’Alessandro et al. 2015, entre otros), Brasil (Amorin Neto y Santos 2005, Leite y Codato 2013, Cardozo 2014, Leite 2015, Marenco 2015, Amorin Neto y Santos 2015, entre otros), México (Loaeza 2005, Alarcón Olguín 2012, Reveles Vázquez 2012, Vidal de la Rosa 2013, Barrientos del Monte 2015, entre otros) y en menor medida, aunque con un desarrollo interesante, Chile (Fuentes y Santana 2005, Rehren y Fernández 2005, Fernández 2005, Viacava 2012, Heiss 2015, Ravecca 2015, entre otros), Uruguay (Garcé 2005, Buquet 2012, Rocha 2012, Garcé y Rocha 2015) y Colombia (Bejarano y Wills 2005, Baquero y Barrero 2013, Barrero y Baquero 2014, Cuellar Argote y Caicedo Ortiz 2015, Leyva y Ramírez 2015, entre otros). Incluso se cuenta actualmente con una reconstrucción no solo de la disciplina, sino de este campo de estudios en sí mismo (Bulcourf et al. 2015) que da cuenta de cómo ha crecido esta línea de investigación en la región, y la conformación del Grupo de Investigación sobre la Historia de la Ciencia Política en América Latina (GIHCIPOLAL) de la Asociación Latinoamericana de Ciencia Política (ALACIP) ha tenido un gran avance con el pronunciamiento del "Manifiesto de Popayán". Todo este desarrollo descriptivo se ha traducido en la ampliación hacia otras vertientes, las cuales se han desprendido de la "típica" lógica de historia e institucionalización de la ciencia política en América Latina, abarcando los temas de género (Rocha Carpiuc 2016), así como cuestionando la idea del rol de la política liberal en la ciencia política latinoamericana ( $\mathrm{Ra}$ vecca 2014, 2015, 2016; Torres-Ruiz y Ravecca 2014), entre otras cosas.

A pesar de estos grandes avances, una de las agendas pendientes más urgentes de esta línea de investigación es la que trata la ciencia política boliviana.

1 Quiero agradecer la colaboración de Diego Murillo (UMSA-ABCP), Marcelo Peralta (UMSA), María Paz Salas (ABCP-UNSLP), Manfredo Bravo (UAGRM), Francisco Parrado (UAP), Carlos Cordero (UMSA-UCB), Jorge Kafka (UMSA), Ariel Benavides (UMSA) y Antonio Mayorga (UMSS) por brindar información para el desarrollo de este trabajo, así como a la evaluación por parte de la revista "Anuario Latinoamericano - Ciencias Políticas y Relaciones Internacionales".

2 El "Manifiesto de Popayán" es un documento elaborado en el marco del III Congreso de Ciencia Política organizado por la Asociación Colombiana de Ciencia Política (ACCPOL) que pretende pronunciarse acerca del devenir de esta línea de estudios y su importancia para la disciplina. 
Dado que la producción sobre la disciplina en el país es escasa, incluso en los trabajos de corte comparativo, no se sabe tanto de Bolivia como de otros países en cuanto a la historia, desarrollo y desafíos que experimenta la disciplina.

La primera aproximación fue elaborada por Marcelo Varnoux (2005) para el número especial de la "Revista de Ciencia Política de la Universidad Católica de Chile". Este trabajo evidencia la cantidad de programas principalmente de licenciatura, las publicaciones que circulan en el país y las asociaciones profesionales, tanto nacionales como regionales, sin embargo existe un desequilibrio en la estructuración del trabajo. A pesar de sus deficiencias esta exploración sirve de insumo para la comparación de Altman (2005) y la de Barrientos del Monte (2012), siendo estos los dos únicos casos, más una pequeña parte del trabajo de Rocha Carpiuc $(2016)^{3}$, donde se toma a Bolivia para las comparaciones regionales.

A pesar de no contar con insumos para pensar e investigar la ciencia política boliviana, esto no significa que sus profesionales y académicos no se preocupen por su historia y su identidad. Dos investigaciones de gran profundidad y rigurosidad han evidenciado el devenir de la ciencia política dentro de las aulas de la Universidad Mayor de San Andrés (UMSA). Primero, Elian Álvarez Gómez (2013) se ocupa de las investigaciones que se realizaron en la carrera de Ciencia Política y Gestión Pública de la UMSA como requisito para la graduación de la carrera y la obtención del título de licenciatura. A partir de ocho líneas de investigación que surgen de la clasificación de ciencias de la UNESCO, la autora da cuenta de dónde se introduce cada una de las 420 investigaciones que se llevaron a cabo entre 1983 y (agosto de) 2013; de igual manera, ordena estos datos de acuerdo a género, periodos ${ }^{4} \mathrm{y}$ modalidad de graduación. ${ }^{5}$

El segundo de los trabajos fundamentales para entender la ciencia política de la UMSA es el de Ramiro Bueno y Gualberto Torrico (2015). Este trabajo se constituye en la principal reconstrucción historiográfica de la carrera y su instituto de investigaciones al mismo tiempo que indaga sobre la producción intelectual propia de la institución publicada en revistas. A lo largo de toda esta investigación, resulta imperante la descripción superficial del contexto político, pero la intimidad del mismo con las etapas de la propia carrera, ya que se toma en cuenta un primer momento de transición democrática equivalente con la posibilidad de desarrollo de la carrera y con paradigmas específicos. Un segundo momento de la carrera se da a la par de la consolidación neoliberal en el país que guía la producción de las revistas de la carrera. Finalmente, el tercer momento se inaugura con la instauración del Estado Plu-

\footnotetext{
3 La autora toma en cuenta programas de pre- y postgrado, revistas científicas y asociaciones profesionales como dimensiones en su investigación; el caso boliviano solamente entra en la última de ellas.

4 La autora reconoce cuatro periodos: 1) de constitución 1983-1992, 2) de modificación 1993-1997, 3) de institucionalización 1998-2009, y 4) de reforma 2010-2013.

5 Se reconocen: tesis de grado, proyecto de grado y trabajo dirigido.
}

Historia y desarrollo de la ciencia política boliviana: una aproximación nacional

Julio Ascarrunz Medinaceli 
Dossier América Latina: desarrollo y balance de la ciencia política rinacional, donde el politólogo trata de formar parte de las amplias y extensas reformas tanto en calidad de gestor como de investigador de dicho proceso (Bueno y Torrico 2015). Más allá del análisis sobre este trabajo, queda clara la "política de la ciencia política" (Ravecca 2014, 2015, 2016) en el país a pesar de no contar con escenarios tan extremos y dicotómicos como son las dictaduras militares vividas en el continente y las democracias liberales (re)instauradas tras esos eventos.

Tras esta breve descripción es que se puede, finalmente, terminar de introducir este trabajo. Dado que la ciencia política boliviana y los estudios de la misma prácticamente no forman parte del concierto internacional, y debido a que los pocos trabajos que tratan la historia y desarrollo de la disciplina en el país están aislados en una Universidad (Álvarez Gómez 2013, Bueno y Torrico 2015) o, en el caso del tratamiento nacional, se encuentran desactualizados (Varnoux 2005), esta investigación tiene como objetivo dar cuenta de la ciencia política en el país a partir de una perspectiva integral, actualizada, y con una ligazón empírica. Para esto es necesario reconstruir la historia integral de la ciencia política en todas sus universidades presentes, revisar la cantidad de publicaciones periódicas y sus problemas, y, por último, entender el proceso de asociación en redes profesionales, sean estas regionales o nacionales.

Para llevar adelante esta tarea, el artículo, en lo que sigue, primero, desarrolla una suerte de marco analítico para el estudio disciplinar, elaborado a partir de la conjunción de diferentes investigaciones ya desarrolladas, sean de caso o comparadas. En una segunda parte se realiza la descripción de las dimensiones señaladas, presentándolas en perspectiva histórica. Un tercer apartado discute sobre la situación actual de la ciencia política boliviana y hace un pequeño esbozo de los desafíos con los que la disciplina (probablemente) tendrá que lidiar si pretende una institucionalización y un desarrollo en la misma línea que sus vecinos de la región. Por último, se presentan las conclusiones del trabajo, así como la agenda de investigación que se debe/puede seguir, tanto para Bolivia como para América Latina.

\section{Marco analítico para el estudio de la ciencia política}

Los muchos trabajos que han abordado la ciencia política en los diferentes países de la región han mostrado tanto ciertas diferencias en los elementos en los que se enfoca, como un cierto consenso sobre algunas dimensiones analíticas. Más allá de dichas (di)similitudes entre esta producción, el presente apartado toma en cuenta, para el desarrollo de su marco analítico, los estudios comparados más amplios (Altman 2005; Barrientos del Monte 2012; Vidal de la Rosa 2013; Bulcourf et al. 2014).

De manera general, el consenso que este campo de estudios ha desarrollado para la construcción de un marco analítico se basa en tres pilares: 1) programas de formación, 2) producción de conocimiento, y 3) redes profesiona- 
les/académicas. Las ponderaciones que usa cada investigador para cada caso difieren entre sí y, en algunos casos, se han aumentado otras variables a los estudios, como vida profesional o competitividad salarial (Altman 2005), ${ }^{6}$ sujetos o actores como constructores de su disciplina (Bulcourf 2012; Bulcourf, Cardozo 2013; Bulcourf et al. 2014), o aspectos teóricos, metodológicos, y empíricos (Vidal de la Rosa 2013).

La dimensión de enseñanza (Altman 2005), comunidad académica y centros de investigación (Barrientos del Monte 2012), o instituciones (Bulcourf et al. 2014) toma en cuenta la cantidad de programas de pre- y postgrado en cada país. Mientras Altman (2005) y Barrientos del Monte (2012) toman la cantidad de programas de licenciatura, maestría y doctorado, ponderándolos con una variable poblacional, Bulcourf et al. (2014) realizan una comparación de corte cualitativo historiográfico entre Argentina, Brasil, y México.

La dimensión de investigación (Altman 2005), revistas académicas (Barrientos del Monte 2012), o producción (Bulcourf et al. 2014) hace un repaso por la producción de conocimiento de los politólogos. Si bien esta dimensión es una constante en todos los trabajos, existen diferencias operativas: por un lado, Barrientos del Monte (2012) hace un recuento de todas las revistas sobre política de la región siempre y cuando cumplan la condición de estar indexadas en Redalyc ${ }^{7}$ y les otorga atributos como el área de conocimiento que tratan y si están indexadas en otros espacios; por otra parte, Altman (2005) y Bulcourf et al. (2014) amplían más el espectro de lo que denominan como investigación, dando lugar al análisis de la existencia de fondos de investigación que sean competitivos e institucionalizados (Altman 2005) o a la descripción de los centros de investigación principales y las obras primordiales de cada caso (Bulcourf et al. 2014).

Por último, la dimensión de comunidad (Altman 2005), asociaciones de politólogos (Barrientos del Monte 2012), o redes (Bulcourf et al. 2014) presenta más homogeneidad al considerarla como la presencia de una asociación nacional que, como actividad principal, organice congresos disciplinares institucionalizados en el tiempo. Sobre este punto, Altman (2005) toma en cuenta la actividad periódica de tales asociaciones con la organización de, por lo menos, un congreso nacional bi-anual y otra actividad anual; Barrientos del Monte (2012), por su parte, menciona el año de creación y hace una reconstrucción histórica de su creación; finalmente, Bulcourf et al. (2014) profundizan más en los tres casos que toman en un sentido historiográfico.

Sobre la primera dimensión, el presente trabajo realiza los dos enfoques revisitados, es decir que se hace tanto la reconstrucción historiográfica como el recuento cuantitativo, el primero sobre la parte histórica y el segundo sobre la actualidad de la dimensión, incorporando a este punto un análisis superfi-

6 Si los politólogos que se dedican (exclusivamente) a la academia a tiempo completo pueden llevar adelante una vida digna.

7 Redalyc es un proyecto para la difusión de la actividad editorial en y sobre Iberoamérica.
Historia y desarrollo de la ciencia política boliviana: una aproximación nacional

Julio Ascarrunz Medinaceli 


\begin{abstract}
Dossier América Latina: desarrollo y balance de la ciencia política

cial de los contenidos mínimos de cada carrera. Sobre la dimensión de investigación se hace un recuento de las revistas existentes en el país y los centros de investigación más conocidos, tanto de manera histórica como reconociendo la situación actual. Por último, sobre la tercera dimensión, se reconstruye la historia de la creación de las distintas redes presentes en el país, pero también se analiza su situación actual. A partir de este ejercicio histórico y contemporáneo es que se pretende dar una pauta del camino a la institucionalización que la ciencia política boliviana debería/podría seguir.
\end{abstract}

\section{Reconstrucción histórica de la ciencia política boliviana}

\section{Programas de formación}

La ciencia política en Bolivia comienza en la Universidad Mayor de San Andrés (UMSA, La Paz) formalmente el año 1983, pero con un par de antecedentes previos a esa creación (Bueno y Torrico 2015). El año 1969, el abogado Alipio Valencia Vega, investigador de los fenómenos políticos desde el derecho político, presenta un proyecto técnico-académico para la creación de la carrera de ciencias políticas en la Facultad de Derecho, sin embargo el proyecto de Valencia no siguió su curso por la irrupción de gobiernos militares "que bajo la Doctrina de Seguridad Nacional, entre otras medidas de control político, intervinieron las universidades" (Bueno y Torrico 2015: 55). Tras el fin del gobierno dictatorial de Hugo Banzer en 1978 se retoma, un año después, la idea de creación de la carrera y el 1 de agosto de 1979 la Comisión de Estructura Académica y Plan de Estudios de la Reunión Sectorial de Facultades de Derecho suscribe la resolución en apoyo a la iniciativa de la UMSA de creación de la carrera de ciencias políticas; no obstante, una vez más, el inicio formal de la carrera se vio interrumpido por el golpe militar liderado por Luis García Meza y Luis Arce Gómez en 1980 (Bueno y Torrico 2015). Es recién en 1983, un año después de la instauración democrática en el país, que la carrera de ciencias políticas entra en funcionamiento de manera definitiva, con la inscripción de 400 estudiantes "como alternativa de estudio a la Carrera de Derecho que en ese momento ya estaba sobrepoblada" (Bueno y Torrico 2015: 61).

Los primeros pasos de la ciencia política en la UMSA estuvieron marcados entre la tuición de la carrera de derecho y las ideas marxistas de sus promotores. Isaac Sandoval, uno de los impulsores de la creación de la carrera, postulaba el perfil del "politicólogo", el cual debía ser un científico y un político, al mismo tiempo que un ideólogo y un revolucionario (Bueno y Torrico 2015), es decir que al principio la carrera fue concebida para la formación de cuadros políticos en la línea marxista. ${ }^{8}$

8 El repaso que hacen Bueno y Torrico (2015) por la propuesta curricular de este primer periodo logra evidenciar fehacientemente esta línea marxista. 
En 1987, la carrera empieza su camino hacia la independencia epistemológica de derecho al incorporar sus primeros docentes politólogos que habían llegado del extranjero junto a profesionales de otras disciplinas como la economía y la filosofía. A pesar de estas incorporaciones, y teniendo ya la primera generación egresada en 1989 con las primeras defensas de tesis a partir de $1990^{\circ}$, es recién en 1992 que se legaliza el funcionamiento de la carrera, la cual hasta ese entonces carecía de presupuesto propio, sus docentes formaban parte de la planilla de derecho, y no podía otorgar títulos profesionales con todas las formalidades legales (Bueno y Torrico 2015). Este hecho se vio sellado con la Resolución no 137/92 del Honorable Consejo Universitario el 27 de agosto de 1992.

Fuera de la UMSA y fuera de la ciudad de La Paz, se crea, también en 1992, la carrera de ciencias políticas en la Universidad Autónoma Gabriel René Moreno (UAGRM) de la ciudad de Santa Cruz de la Sierra a partir de un proyecto elaborado en 1991 por el economista José Ortiz Mercado, el cual fue enriquecido por Isaac Sandoval, en especial para la creación de la primera malla curricular. ${ }^{10}$ Tras la creación mediante Resolución del Ilustre Consejo Universitario (ICU) de la UAGRM el 13 de agosto de 1992, la carrera inicia sus actividades en septiembre del mismo año y logra su primera promoción de egresados en 1996, la cual constaba de un grupo de "12 estudiantes de los 93 que ingresaron en septiembre de 1992" (Carrera de Ciencia Política y Administración Pública 2015: 2).

El periodo entre 1996 y 1997 significó un hito importante para la ciencia política boliviana. La primera generación de la UAGRM empezaba a dibujar la institucionalidad de la disciplina en esta universidad del oriente boliviano y ese mismo año se producía una reforma al plan de estudios en la UMSA, la cual significó un aumento en las materias impartidas propias de la ciencia política de un 21,1\% en la malla curricular de 1986 a un $41 \%$ en la de 1996 (Bueno y Torrico 2015). Por otra parte, en 1997, en la ciudad de Cochabamba, en la Universidad Mayor de San Simón (UMSS), se creaba el programa de ciencias políticas dentro de las carreras de derecho, sociología y economía en la Facultad de Ciencias Jurídicas y Políticas (Flores 2014; Aspi 2016). No obstante este paso importante en la ciencia política cochabambina, no es hasta el año 2001 que la carrera se independizó completamente de sus pares disciplinares y logró autonomía como carrera mediante la Resolución 20/01 del Honorable Consejo Universitario de la UMSS.

Con estas tres universidades se completaba el eje troncal del país (La Paz, Cochabamba y Santa Cruz) con carreras de ciencia política en sus universidades públicas. A fines del siglo XX, de igual manera, se inauguraba una "oleada"

9 Los primeros egresados, en orden respectivo, fueron Grace Ivana Deheza, Julio Ballivián, Moira Zuazo, Jimena Costa, Hipólito Encinas, Jorge Kafka, Marcelo Varnoux, Julio Velásquez, Hugo Vega y Marco Antonio Saavedra (Bueno, Torrico 2015).

${ }^{10}$ Datos provistos por Manfredo Bravo, exdirector de la carrera.
Historia y desarrollo de la ciencia política boliviana: una aproximación nacional

Julio Ascarrunz Medinaceli 
Dossier

América Latina: desarrollo y balance de la ciencia política

de carreras de ciencias políticas en las universidades privadas de la ciudad de La Paz. La primera de ellas fue la Universidad Nuestra Señora de La Paz (UNSLP) que en 1999 creó la Facultad de Ciencias Políticas y Sociales ${ }^{11}$; el año 2000 se crea la carrera de ciencias políticas y filosofía en la Universidad de la Cordillera, sin embargo la carrera, junto con toda la universidad, tuvo que cesar sus funciones el año $2013^{12}$; por último, la Universidad Católica Boliviana (UCB) sede La Paz, el año 2003, crea una serie de carreras de pregrado entre las que se encontraba la de ciencias políticas.

En estos últimos años, otras dos universidades públicas del país se adentraron en la fundación de carreras de ciencia política. Primero, el año 2008, la Universidad Amazónica de Pando (UAP) de dicho departamento y con sede en la ciudad de Cobija funda el programa de ciencia política y reformula su malla curricular el 2012, sin embargo, como la mayor parte de la oferta académica de la UAP, el programa de ciencia política termina formando más estudiantes con títulos técnicos, medio y superior ${ }^{13}$. Por último, el año 2011 se crea la carrera de ciencias políticas en la Universidad Pública de El Alto (UPEA) mediante Resolución No 23/2011 del Honorable Consejo Universitario y refrendado por el Comité Ejecutivo de la Universidad Boliviana (CEUB) ${ }^{14}$ en diciembre de 2012 mediante Resolución No 23/2012, hecho que destaca dado

Gráfico 1.

Creación de carreras de ciencia política en línea de tiempo

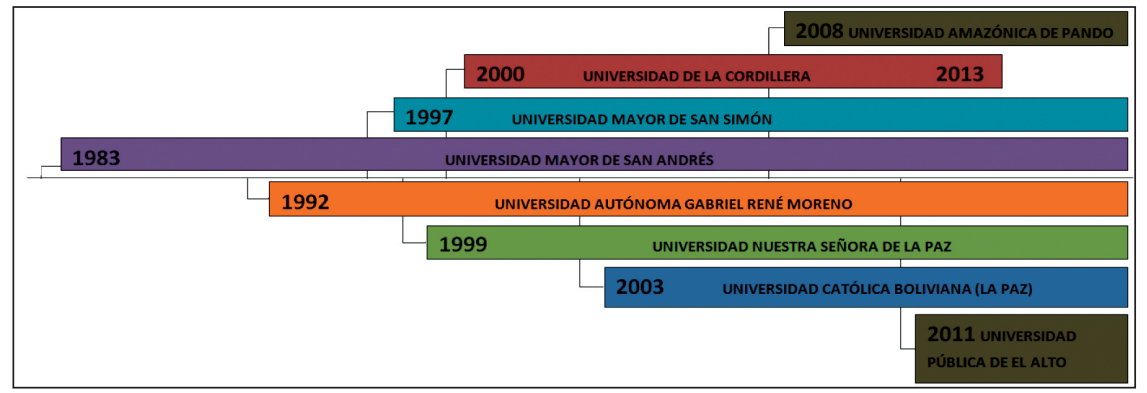

Fuente: elaboración propia.

${ }^{11}$ Información provista por María Paz Salas, actual presidenta de la Asociación Boliviana de Ciencia Política, exdocente de la UNSLP y parte de la primera generación de politólogos de dicha universidad.

12 Información provista a finales del año 2016 por el Área de Información Académica de dicha universidad. Actualmente la descripción de la carrera, así como los requisitos para su ingreso, se encuentran en la página web de la universidad: https://www.ucordillera.edu.bo/index. php/ciencias-politicas-y-filosofia UAP.

13 Información provista por Francisco Parrado, graduado de la UMSA y actual docente de la

${ }^{14}$ El CEUB es el organismo encargado de la coordinación, planificación y programación de las actividades que realiza la Universidad Boliviana, la cual está compuesta por todas las universidades públicas autónomas del país más la Universidad Andina Simón Bolívar, la Universidad Policial “Mcal. Antonio José de Sucre”, la Universidad Católica Boliviana y la Escuela Militar de Ingeniería. 
que la creación de la universidad data del año 2000 y la obtención de autonomía universitaria del 2005.

El gráfico 1 resume el recorrido histórico de la ciencia política boliviana a partir de fechas y logra dar un panorama completo de lo descrito anteriormente. Se puede apreciar los tres momentos de la ciencia política boliviana donde, primero, destaca la creación en las principales universidades públicas del país entre 1983 y 1997; una segunda etapa marcada por la irrupción de universidades privadas de la ciudad de La Paz en la oferta académica politológica, dada entre 1999 y 2003; y, por último, la tercera etapa caracterizada por la búsqueda de protagonismo de universidades públicas de menor trayectoria temporal como la UAP y la UPEA. Estas ocho universidades conforman la formación inicial de ciencia política (estricta ${ }^{15}$ ) en el país.

En cuanto a la oferta académica de posgrado, la ciencia política boliviana ha sido irregular y dependiente. El programa de maestría más reconocido se encuentra en el Postgrado en Ciencias del Desarrollo (CIDES) de la UMSA, siendo una oferta combinada de filosofía y ciencia política. Otra oferta, exclusiva de la disciplina, es de la Unidad de Postgrado y Relaciones Internacionales de la Facultad de Derecho y Ciencias Políticas de la UMSA, la cual en 2010 y en 2015 presentó su programa. Se han llevado a cabo otros programas de maestría que están relacionados a la ciencia política, pero no son exclusivos de la disciplina, como por ejemplo la Maestría de Investigación en Ciencias Sociales para el Desarrollo, en sus versiones 2004, 2005, 2007 y 2009, de la Universidad de Postgrado para la Investigación Estratégica en Bolivia (UPIEB); mientras que otras ofertas son enfocadas a la gestión pública y por parte de universidades que no cuentan con la licenciatura ni en ciencia política ni en administración pública de manera independiente, como por ejemplo la Escuela Militar de Ingeniería (EMI) o la Universidad de Aquino Bolivia (UDABOL). En cuanto a doctorados, la UMSA ha ofertado un programa en gestión del desarrollo y políticas públicas el año 2010 que no ha tenido mucha más continuidad.

Como se puede apreciar fácilmente, la formación posgradual politológica en Bolivia sufre de dependencia de otras disciplinas como la filosofía o el derecho, o está inserta en programas más amplios de ciencias sociales; de igual manera, la mayor parte de los programas no se encuentran institucionalizados por lo que no tienen continuidad en el tiempo.

15 Se habla de una formación estricta dado que si se cuentan las carreras de relaciones internacionales que están separadas de la formación en ciencia política, la cantidad sube de ocho a trece, es decir que existen cinco carreras en todo el país de las cuales solamente la que está en la UAGRM comparte casa de estudios con una carrera de ciencia política, lo cual deja otras cuatro licenciaturas en relaciones internacionales en universidades privadas y completamente separadas de la disciplina.
Historia y desarrollo de la ciencia política boliviana: una aproximación nacional

Julio Ascarrunz Medinaceli 
Dossier América Latina: desarrollo y balance de la ciencia política

\section{Producción de conocimiento}

La historia de las revistas y centros de investigación en ciencia política es irregular y poco conocida, donde solamente tres de las ocho carreras cuentan tanto con centros de investigación y publicaciones, y otras dos solamente con un instituto o una publicación. El primer centro es el Instituto de Investigaciones en Ciencia Política (IINCIP) de la UMSA que tiene su creación en 1992 a partir de un proyecto presentado por Isaac Sandoval (Bueno y Torrico 2015), con el objetivo de llevar adelante tareas de investigación, tanto de docentes como de estudiantes, que ya habían tenido sus primeras experiencias en las tesis defendidas hasta la época y la publicación de una revista institucional. La primera experiencia editorial de la UMSA se llevó a cabo con "Ensayo, Revista de teoría y análisis político" en 1989, 1990, y 1991, la cual fue sustituida por la "Revista Ciencia Política" en 1994. A partir de dicha sustitución, la revista de la carrera tuvo ejemplares los años 1996, 1997, 1999, 2002, 2003, 2007, 2008, 2009, 2010, 2011, y 2012 (Bueno y Torrico 2015).

Por su parte, la UAGRM ha incursionado en la institucionalización de la investigación recién a partir de 2006 con la creación del Centro Regional de Investigación en Ciencias Políticas y Sociales, y su experiencia editorial se ha visto concretada con la revista "Conocimiento y Política" el año $2008^{16}$ y refrendada a partir de la publicación facultativa "Criterio Académico" desde 2014. La UMSS cuenta con el Centro de Investigaciones de Ciencia Política creado el año 2005 que acoge a la revista "Estudios Políticos", la cual se ha desarrollado desde el año 2009 de manera anual. ${ }^{17}$

Por último, la carrera de ciencias políticas de la Facultad de Ciencias Políticas y Sociales de la UNSLP cuenta con la revista "Análisis Político" desde $1997^{18}$ de manera semestral por dos años (97-98, números 1 al 4), de manera anual por los siguientes cuatro años (99-2002, números 5 al 8), y con una periodicidad más irregular desde entonces pero llegando hasta el número 17. A pesar de esto, la UNSLP no cuenta con un centro de investigaciones propio de la carrera de ciencia política ni afín. Un escenario contrario muestra la UCB sede La Paz, que sí cuenta con el Instituto para la Democracia dependiente de la Facultad de Ciencias Jurídicas y Políticas, pero no tiene una publicación periódica; ahora bien, el caso del Instituto para la Democracia de la UCB, si bien no es un centro exclusivo en ciencia política, contiene una de las temáticas centrales de la disciplina.

16 Manfredo Bravo Chávez, exdirector de la carrera de ciencia política y administración pública de la UAGRM, menciona que hasta la fecha se han publicado solamente 4 números.

17 Información provista por Antonio Mayorga Ugarte, docente de la UMSS.

18 Nótese que la revista data de dos años antes que la creación de la Facultad. 


\section{Redes politológicas}

La conjunción de los politólogos bolivianos ha sido y es un proceso dificultoso, que se puede clasificar en dos conjuntos. El primero de ellos es el de la creación de las redes departamentales fuera de la ciudad de La Paz que han intentado ser una respuesta representativa de los profesionales en ciencia política que están más allá de la sede de gobierno. Así, primero, en 1996, se crea el Colegio Departamental de Politólogos de Santa Cruz (CDPSC), el cual no ha tenido mucha actividad ni influencia en estos años y a partir de 2008 funciona de manera dividida (Entrevista a Manfredo Bravo). Posteriormente, en julio de 2002, se crea el Colegio Departamental de Politólogos de Cochabamba (CDPC). Ambas asociaciones departamentales han estado muy ligadas a las respectivas universidades públicas que contienen la carrera de ciencia política en sus territorios.

El segundo conjunto se posiciona dentro de la ciudad de La Paz. El año 1998 se funda el Colegio Departamental de Politólogos de La Paz (CDPLP) a partir de una iniciativa conjunta entre docentes y estudiantes de la UMSA que veían en la política universitaria las puertas cerradas a su representación e intereses. ${ }^{19}$ Siguiendo la cronología de los eventos, en febrero de 2002 se crea la Asociación Boliviana de Ciencia Política (ABCP) de una manera similar que el CDPLP. Las mismas lógicas políticas que habían permeado la universidad en 1998 llegaron a este colegio profesional, lo cual causó que una parte de sus miembros se decidan por la separación y se aventuren a fundar la ABCP. ${ }^{20} \mathrm{De}$ igual manera, y más allá de las pugnas políticas internas dentro de cada ámbito, se reconoce la diferencia de que el colegio fue concebido con una lógica de representación de intereses gremiales y corporativos de los profesionales de la ciencia política sea que éstos estén ligados o no a la academia, mientras que la $\mathrm{ABCP}$ pregonaba un desarrollo científico de la disciplina. Esta situación de disconformidad y desprendimientos continuos que llevó a la creación de la ABCP por fuera del CDPLP derivó en la decisión de que la membrecía de la Asociación sea por invitación directa de personalidades destacadas en el ámbito político y politológico a partir de la aprobación del directorio.

El motivo por el que se realiza la separación en dos conjuntos no es una afirmación del centralismo paceño imperante en la vida política y académica del país, sino que responde a una lógica de interconexión entre las distintas redes de politólogos (formales) que existen en Bolivia. Mientras que el primer conjunto tiene la característica de desconectar su historia fundacional entre sí, el segundo conjunto claramente muestra que las actividades de una red produjeron la creación y estructura de la otra. Sin embargo, ambos grupos

${ }_{19}$ Información provista por Marcelo Peralta, actual director de la Carrera de Ciencia Política y Gestión Pública de la UMSA.

${ }^{20}$ Información provista por Diego Murillo, exdirector de la Carrera de Ciencia Política y Gestión Pública de la UMSA.
Historia y desarrollo de la ciencia política boliviana: una aproximación nacional

Julio Ascarrunz Medinaceli 
Dossier América Latina: desarrollo y balance de la ciencia política demuestran, de una u otra manera, la estrecha conexión de estas redes a las universidades, en especial a las públicas.

Por último, cabe señalar que, a diferencia de la creencia generalizada que afirma que la ciencia política boliviana no ha tenido congresos de ciencia política, hay algunas experiencias que demuestran lo contrario y, al mismo, tiempo dan lecciones. Como bien detallan Bueno y Torrico (2015), se llevaron a cabo dos congresos de ciencia política, uno en 1999 y otro en 2003, pero con otros antecedentes más en 1998, 2009 y 2010, aunque con características diferentes. ${ }^{21}$ La primera experiencia en 1998 fue una reunión sectorial de las carreras de ciencias políticas del sistema universitario donde participaron las universidades de San Andrés, San Simón y Gabriel René Moreno. Este primer acontecimiento, si bien no fue de corte académico, mostró los primeros pasos, o al menos la posibilidad de los mismos, en el camino de la integración de la ciencia política boliviana.

No fue hasta 1999 que se llevó a cabo el primer congreso (académico) de ciencia política en Bolivia, donde estuvieron como invitados de honor los profesores Dieter Nohlen y Arend Lijphart. El organizador del congreso fue Carlos Cordero quien, en ese entonces, además de ser docente de la UMSA, trabajaba en el Programa de Gobernabilidad de la Vicepresidencia; dado este cargo, Cordero contrató a Nohlen y a Lijphart para la realización de una consultoría en el marco del programa y aprovechó la presencia de ambos en el país para realizar un congreso alrededor de su llegada.

Los dos siguientes congresos, realizados en 2003 y 2009, no fueron tan llamativos como el primero, aunque siguieron dando la lección de que sí era posible la realización de estos eventos en el país, si se juntaba la voluntad de las instituciones y las personas al mando de las mismas. La última experiencia que juntó a las carreras de ciencia política del país fue la reunión sectorial de 2010, llevada a cabo en Santa Cruz. Además de las tres universidades que participaron de la primera sectorial, se sumaron la UCB y la UAP que ya contaban con sus programas de ciencia política.

\section{Discusión}

De manera integral, a partir de este (breve) repaso histórico, se puede apreciar que la ciencia política boliviana solamente tiene robustez institucional en la formación de pregrado, incluso con las debilidades que significa una dependencia, aunque reducida en relación al pasado, de otras disciplinas, en especial del derecho. El hecho de que la mayoría de las carreras estén insertas en facultades de derecho y que muchas otras facultades de derecho de universidades públicas del país contemplen la formación en ciencias políticas junto a

${ }^{21}$ Información provista por Carlos Cordero, docente e investigador en ciencia política en la UMSA y la UCB. 
la abogacía y la otorgación de un título combinado ${ }^{22}$, es una primera muestra de esa dependencia en pregrado.

Por otra parte, la disciplina en Bolivia se ha desconectado de áreas propias como lo son las relaciones internacionales y las políticas públicas. Las relaciones internacionales, por una parte, y a nivel de pregrado, han realizado un esfuerzo por independizarse en cuanto a su formación no solamente fuera de las carreras, sino también fuera de las universidades que ofrecen licenciaturas en ciencia política. El área de políticas públicas, en cambio, ha transitado más hacia la economía y el derecho, en especial en la oferta de postgrado donde se puede observar la presencia de universidades y programas de gestión y políticas públicas dependientes de unidades académicas propias de estas disciplinas en lugar de la ciencia política, e incluso con un plantel docente mayoritariamente economista.

La investigación politológica encuentra su mayor problema no en la falta de producción y/o difusión, sino en los alcances de esos aspectos. La presencia de revistas de varias unidades académicas presentes desde la década de los 80 del siglo pasado es una buena señal de la producción de sus académicos, sin embargo, estas publicaciones luchan con la informalidad y la falta de cumplimiento de estándares científicos reconocidos y aceptados internacionalmente. Basta con revisar los dos principales portales de revistas científicas en América Latina, Redalyc y Scielo, para evidenciar que ninguna de las revistas señaladas en este recorrido está, ni ha estado, indexada en dichos portales; la aproximación más cercana está en la revista “T’inkazos”, del Programa de Investigación Estratégica en Bolivia (PIEB), la cual es una revista de ciencias sociales, de manera general, donde algunos de los/as politólogos/as más reconocidos/as han publicado algunos de sus trabajos. Otra porción de evidencia acerca de la informalidad de las revistas politológicas del país se puede observar en su periodicidad y condiciones para la publicación: primero que ninguna de ellas cumple con una periodicidad clara y se terminan publicando número de dichas revistas de manera anual, semestral, bianual, y hasta trianual; y, segundo, que ninguna de estas empresas editoriales tienen una convocatoria de artículos ni una referencia imparcial para decidir cuáles artículos entran y cuáles no.

Por último, la situación de las asociaciones sigue siendo la misma de cuando empezaron. Los tres colegios de politólogos, sean de Santa Cruz, de Cochabamba, o de La Paz, funcionan con mucha irregularidad producto de una falta de institucionalidad clara y con presencia meramente regional. Si bien en algún momento estas asociaciones tocan temas de interés nacional, como algún proceso electoral general, la mayor parte de su actividad y "público" están enmarcados dentro de su propio departamento. Por último, la ABCP, a pesar de las intenciones y acciones desarrolladas en estos 15 años de vida institucional, no ha podido terminar de consolidar una institucionalidad fuerte

22 Por ejemplo la Autónoma "Juan Misael Saracho" de Tarija, la Técnica de Oruro, o la Mayor San Francisco Xavier de Chuquisaca, entre otras.
Historia y desarrollo de la ciencia política boliviana: una aproximación nacional

Julio Ascarrunz Medinaceli 
Dossier América Latina: desarrollo y balance de la ciencia política

producto del fantasma de la politización interna, es decir que las motivaciones que llevaron a crear una asociación selectiva en cuanto a sus miembros en 2002 siguen presentes en la actualidad, lo que provoca la falta de legitimidad que esta red tiene en toda la comunidad politológica del país. En definitiva, la comunidad politológica boliviana no existe en una sola red y se divide entre situaciones regionales, posturas políticas, posiciones académicas y actividades profesionales.

La ciencia política en Bolivia ha tenido avances importantes, en especial en relación a aspectos cuantitativos de la enseñanza de pregrado, sin embargo el camino hacia la institucionalización, en el mismo sentido que sus vecinos regionales, es largo y los retos son varios. Primero, es necesario que la formación académica traspase las fronteras del pregrado y logre posicionar programas de maestría y doctorado exclusivos de la disciplina que sean estables en el tiempo; esto puede empezar a vislumbrar su independencia de otras disciplinas de las ciencias sociales pero con un alto rendimiento y capacitación académica. Segundo, resulta imperante que la disciplina logre posicionar sus revistas, o crear nuevas, de manera que cumplan los estándares mínimos de calidad científica y se puedan posicionar en el escenario regional y mundial, así insertarse en el diálogo científico global en lugar de cerrarse dentro de sus fronteras. Por último, las redes no necesariamente tienen el deber de juntarse dentro de una sola, esta pluralidad de comunidades tiene objetivos distintos y permite la representación descentralizada de los profesionales en ciencia política, sin embargo, sí es preciso que estas asociaciones logren obtener una mayor institucionalidad y puedan abrir sus puertas al creciente mundo de politólogos.

\section{Conclusiones}

El repaso realizado en el presente trabajo ha servido para reconstruir la ciencia política en Bolivia y poder aportar tanto para el conocimiento interno de esta disciplina en el país como para completar el mapa de este campo de estudios de la región. Las características descriptivas, casi exploratorias, de este artículo hacen que se abran más preguntas en lugar de encontrar respuestas, y así se inaugura una línea de investigación rica para los politólogos bolivianos interesados en su propia historia, al mismo tiempo que otorga insumos para la reflexión y el estudio en la región.

Los hallazgos aquí presentados tienen un corte más endógeno a la propia disciplina boliviana, pero a partir de esta aproximación nacional es que es posible continuar la construcción de conocimiento e indagar sobre la relación entre la producción intelectual politológica con su entorno político con trabajos de corte bibliométrico, por ejemplo. Es importante, también, señalar que desde la recolección de datos para el presente trabajo ${ }^{23}$ ha habido varios hechos de

\footnotetext{
${ }^{23}$ El trabajo fue elaborado entre finales de 2016 y principios de 2017.
} 
"crecimiento" de la disciplina en el país, por mencionar algunos: se ha abierto un Doctorado en Ciencia Política y Relaciones Internacional en la UMSA con plantel docente nacional e internacional; se ha publicado el primer número de la Revista Boliviana de Ciencia Política; y se ha fundado el Colegio de Politólogos de Tarija. Estos nuevos hechos alteran positivamente el diagnóstico de la disciplina que puede ser revisitado en la continuación de esta línea de investigación.

De igual manera, la experiencia boliviana ha dado luces sobre otra posible concepción de la política de la ciencia política distinta a la expuesta por Ravecca $(2014,2015,2016)$, es decir que es necesario discutir sobre las pugnas políticas que existen al interior de las universidades y/o asociaciones y sus implicancias sobre el desarrollo de la disciplina. De igual manera, resulta interesante preguntarse sobre una posible cultura académica y su impacto sobre esta $\mathrm{u}$ otra disciplina. Las oportunidades son variopintas y solamente dependen de los intereses y curiosidades de cada investigador para llevarse a cabo.

\section{Bibliografía}

Alarcón Olguín V. (2012), La ciencia política mexicana. Reflexiones sobre su pasado, presente y porvenir, "Política. Revista de Ciencia Política", vol. 50, no 1, pp. 31-57.

Altman D. (2005), La institucionalización de la ciencia política en Chile y América Latina: una mirada desde el sur, "Revista de Ciencia Política", vol. 25, no 1, pp. 03-15.

Álvarez Gómez E. (2013), El estado de la investigación en la ciencia política. Memoria de tesis de grado, proyecto de grado y trabajo dirigido. Instituto de Investigaciones en Ciencia Política de la Carrera de Ciencia Política y Gestión Pública de la Universidad Mayor de San Andrés, La Paz.

Amorim Neto O., Santos F. (2005), La ciencia política en Brasil: el desafío de la expansión, "Revista de Ciencia Política", vol. 25, no 1, pp. 101-110.

Amorim Neto O., Santos F. (2015), La ciencia política en Brasil en la última década: La nacionalización y la lenta superación del parroquialismo, "Revista de Ciencia Política", vol. 35, no 1, pp. 19-31.

Aspi J. (diciembre 2016), XV Aniversario de la Carrera de Ciencia Política [Entrada en blog], disponible en: https://cienciapoliticaumss.blogspot.com/2016/12/xv-aniversario-de-lacarrera-de-ciencia.html

Baquero S.A., Barrero Escobar F. (2013), La ciencia política y sus métodos. Una comparación de los programas de ciencias sociales en Colombia, "Revista Co-herencia", vol. 10, no 19, pp. 207-234.

Barrero F., Baquero S. A. (2014), Los retos de la ciencia política en Colombia, "Iberoamericana", vol. XIV, no 56, pp. 191-195.

Barrientos del Monte F. (2012), La institucionalización de la ciencia política en América Latina, en: F. Reveles Márquez (coord.), La ciencia politica en México hoy: ¿qué sabemos?, Universidad Nacional Autónoma de México, México.

Barrientos del Monte F. (2015), Crecimiento e institucionalización de la ciencia política en México, "Revista de Ciencia Política", vol. 35, no 1, pp. 95-120.
Historia y desarrollo de la ciencia política boliviana: una aproximación nacional

Julio Ascarrunz Medinaceli 
Dossier América Latina: desarrollo y balance de la ciencia política
Bejarano A. M., Wills M. E. (2005), La ciencia política en Colombia: de vocación a disciplina, "Revista de Ciencia Política", vol. 25, no 1, pp. 111-123.

Bueno R., Torrico G. (2015), Ciencia Política Académica. Trayectoria histórica y política de la Carrera de Ciencia Política y Gestión Pública (1983-2012), Carrera de Ciencia Política y Gestión Pública de la Universidad Mayor de San Andrés, La Paz.

Bulcourf P. (2012), El desarrollo de la ciencia política en Argentina, "Política. Revista de Ciencia Política", vol. 50, no 1, pp. 59-92.

Bulcourf P., Cardozo N. (2013), La Ciencia Política en la Argentina: su desarrollo e institucionalización, "Revista Debates", vol. 7, no 3, pp. 57-88.

Bulcourf P., Cardozo N., Campos M. (2013), Los estudios internacionales en la Argentina en perspectiva histórica, "Revista Aportes para la Integración Latinoamericana", año 19, no 29, pp. 31-64.

Bulcourf P., Gutiérrez Márquez E., Cardozo N. (2014), El desarrollo de la ciencia política en Argentina, Brasil y México: construyendo una mirada comparada, "Anuario Latinoamericano - Ciencias Políticas y Relaciones Internacionales”, vol. 1, pp. 155-184.

Bulcourf P., Gutiérrez Márquez E., Cardozo N. (2015), Historia y desarrollo de la ciencia política en América Latina: reflexiones sobre la constitución del campo de estudios, "Revista de Ciencia Política", vol. 35, no 1, pp. 179-199.

Buquet D. (2012), El desarrollo de la ciencia política en Uruguay, "Política. Revista de Ciencia Política", vol. 50, no 1, pp. 5-29.

Cardozo N. (2014), La ciencia política en Brasil: una historia en pujante desarrollo, "Iberoamericana", vol. XIV, no 56, pp. 186-190.

Carrera de Ciencia Política y Administración Pública (2015), Plan de Desarrollo Estratégico 2015-2020, Universidad Autónoma Gabriel René Moreno, Santa Cruz.

Cuellar Argote J. A., Caicedo Ortiz J. A. (2015), ¿Hacia dónde va la ciencia política?: reflexiones sobre la disciplina en Colombia, Universidad del Tolima, Ibagüé.

D’Alessandro M., Abal Medina J., Leiras, M. (2015), La ciencia política en Argentina 20052014: El camino de la consolidación dentro y fuera de las aulas universitarias, "Revista de Ciencia Política", vol. 35, no 1, pp. 3-17.

Fernández M. A. (2005), Ciencia política en Chile: un espejo intelectual, "Revista de Ciencia Política", vol. 25, no 1, pp. 56-75.

Flores L. (Enero 2014), Reseña histórica [Entrada en blog], disponible en: http:// cienciaspoliticas-umss.blogspot.com/p/resena-historica.html

Fuentes C., Santana G. (2005), El "boom" de la ciencia política en Chile: escuelas, mercado y tendencias, "Revista de Ciencia Política", vol. 25, no 1, pp. 16-39.

Garcé A. (2005), La ciencia política en Uruguay: un desarrollo tardío, intenso y asimétrico, "Revista de Ciencia Política", vol. 25, no 1, pp. 232-244.

Garcé A., Rocha Carpiuc C. (2015), La ciencia política en Uruguay: Entre la profesionalización, la partidización y el fantasma del "Movimiento Perestroika", "Revista de Ciencia Política", vol. 35 , no 1 , pp. 121-144.

Heiss C. (2015), Ciencia política en Chile: ¿Una disciplina consolidada?, "Revista de Ciencia Política", vol. 35, no 1, pp. 47-70. 
Leiras M., Abal Medina J., D’Alessandro M. (2005), La ciencia política en Argentina: el camino de la institucionalización dentro y fuera de las aulas universitarias, "Revista de Ciencia Política", vol. 25, no 1, pp. 76-91.

Leite F., Codato A. (2013), Autonomização e institucionalização da Ciência Política brasileira: o papel do sistema Qualis-Capes, "Revista de Discentes de Ciência Política da UFSCAR", vol. 1, no 1, pp. 1-21.

Leite F. (2015), O campo de produção da Ciência Política brasileira contemporânea: uma análise histórico-estrutural de seus princípios de divisão a partir de periódicos, áreas e abordagens, Tesis presentada para la obtención del grado de Doctor en Sociología por la Universidad Federal de Paraná.

Leyva S., Ramírez M. F. (2015), La ciencia política en Colombia: Una disciplina en continua expansión, "Revista de Ciencia Política", vol. 35, no 1, pp. 71-94.

Loaeza S. (2005), La ciencia política: el pulso del cambio mexicano, "Revista de Ciencia Política", vol. 25, no 1, pp. 192-203.

Marenco A. (2015), When Institutions Matter: CAPES and Political Science in Brazil, "Revista de Ciencia Política”, vol. 35, no 1, pp. 33-46.

Ravecca P. (2014), La política de la ciencia política en Chile y Uruguay: primeros hallazgos de una agenda de investigación, Instituto de Ciencia Política de la Facultad de Ciencias Sociales de la Universidad de la República, Documento de Trabajo On Line no (01/14), http://209.177.156.169/libreria_cm/archivos/pdf_578.pdf, fecha de consulta: 15.12.2016.

Ravecca P. (2015), Our discipline and its politics. Authoritarian political science: Chile 19791989, "Revista de Ciencia Política", vol. 35, no 1, pp. 145-178.

Ravecca P. (2016), The comparative politics of political science: Chile and Uruguay in authoritarian times, "Revista Andina de Estudios Políticos", vol. VI, no 1, pp. 4-17.

Rehren A., Fernández M. (2005), La evolución de la ciencia política en Chile: un análisis exploratorio (1980-2000), "Revista de Ciencia Política", vol. 25, no 1, pp. 40-55.

Reveles Vázquez F. (2012), La ciencia política en México hoy: ¿qué sabemos?, Facultad de Ciencias Políticas y Sociales de la Universidad Nacional Autónoma de México (UNAM), México, D.F.

Rocha Carpiuc C. (2012), El desarrollo de la ciencia politica en Uruguay (1987-2009). Temas, teorías y metodologías, Licenciatura de Ciencia Política, Facultad de Ciencias Sociales, Universidad de la República, Montevideo.

Rocha Carpiuc, C. (2016), Women and diversity in Latin American political science, "European Political Science", vol. 15, no 4, pp. 457-475, DOI: 10.1057/s41304-016-0077-4

Torres-Ruiz A., Ravecca P. (2014), The politics of Political science and toxic democracies. A hemispheric perspective, "Crítica Contemporánea. Revista de Teoría Política", no 4, pp. $107-135$.

Varnoux M. (2005), La ciencia política en Bolivia: entre la reforma política y la crisis de la democracia, "Revista de Ciencia Política", vol. 25, no 1, pp. 92-100.

Viacava Gatica J. (2012), La ciencia política en Chile: una carrera en expansión y transformación, "Política. Revista de Ciencia Política", vol. 50, no 1, pp. 93-110.

Vidal de la Rosa G. (2013), Ensayos sobre la ciencia política en México y Latinoamérica, Universidad Autónoma Metropolitana - Azcapotzalco, México D.F.
Historia y desarrollo

de la ciencia política boliviana: una aproximación nacional

Julio Ascarrunz Medinaceli 